\title{
Venetoclax and Azacytidine in Relapsed/Refractory Acute Myeloid Leukemia and High Risk Myelodysplastic Syndrome: Bursa Uludag University Experience
}

\author{
İbrahim Ethem PINAR ${ }^{1}$, Fahir ÖZKALEMKAŞ ${ }^{1}$, Vildan ÖZKOCAMAN ${ }^{1}$, Tuba ERSAL ${ }^{1}$, Cumali \\ YALÇIN $^{1}$, Bedrettin ORHAN ${ }^{1}$, Ömer CANDAR ${ }^{1}$
}

${ }^{1}$ Bursa Uludağ University Medical Faculty, Department of Internal Medicine, Division of Hematology, Bursa, Turkey

Turk J Int Med 2021;3(Supplement 1):S42-S43

DOI: $10.46310 /$ tjim. 877068

Keywords: Venetoclax, azacitidine, acute myeloid leukemia, myelodysplastic syndrome

Acute myeloid leukemia (AML) is the deadliest leukemia in adults, with a mean age of 67 years at the time of diagnosis. ${ }^{1,2}$ Approximately 2050 percent of patients do not achieve a complete remission with intensive induction chemotherapy, and most patients' relapse. ${ }^{3}$ Azacytidine, one of the hypomethylating agents, is commonly used to control the disease. Venetoclax is a BCL-2 inhibitor that is synergistic with azacytidine. The addition of venetoclax to the treatment is based on a randomized trial that improves survival and increases response rates. ${ }^{4,5}$ This study aims to evaluate the efficacy and safety data of venetoclax+azacytidine combination, a new treatment modality in relapsed/refractory $(R / R)$ AML and high-risk myelodysplastic syndrome (MDS).

$\mathrm{R} / \mathrm{R} A M L$ and high-risk MDS patients aged $\geq 18$ years were included in the study. The off-label use of drugs for each patient was received permission from the Turkish Medicines and Medical Devices
Agency. Venetoclax was given orally on day 1: $100 \mathrm{mg}$, day 2: $200 \mathrm{mg}$, next days: $400 \mathrm{mg} /$ day. Azacytidine 1-7. days (every 28 days) $75 \mathrm{mg} / \mathrm{m}^{2}$ administered subcutaneously (alternatively, 50 $\mathrm{mg} / \mathrm{m}^{2}$ as 5-2-5). All patients received prophylaxis for tumor lysis syndrome (TLS) for at least three days. In patients receiving antifungal therapy that inhibits CYP3A enzyme activity, the dose of venetoclax was reduced by $75 \%$.

A total of 15 patients, $14 \mathrm{R} / \mathrm{R}$ AML and $1 \mathrm{MDS}$ RAEB-1, followed in the Bursa Uludag University Hematology Department was included in the study. $60 \%(n=9)$ of these patients were female, and the median age was 65 years (32-84). 50\% $(n=7)$ of AML patients were de novo leukemia. Fourteen patients were between ECOG 0 and 2. Fourteen were in the medium, and one was in the high cytogenetic risk group. The treatment indication was salvage therapy in 13 patients and consolidation in 2 patients. Median 2 cycles (14) of venetoclax+azacytidine chemotherapy was

Received:February 10, 2021;Accepted:March 5, 2021;Published Online:March 6, 2021 
applied to the patients. The median follow-up time was 1.2 months (0.1-6.5). The median time to first response with treatment was one month $(0.5-5)$. The refractory disease was persistent in all four patients who were found to have a refractory disease at the end of the first cycle. The best responses obtained with venetoclax+azacytidine were complete remission (CR) in one patient, complete remission with incomplete hematologic recovery (CRi) in one patient, morphological leukemia-free state (MLFS) in 4 patients, and partial response $(\mathrm{PR})$ in one patient. The remission duration in 2 patients with composite remission (CR + CRi) was 3.5 and 17 months. Minimal residual disease was negative in only one patient. Venetoclax+azacytidine was used as a bridge therapy to allogeneic transplant in one patient. With Venetoclax+azacytidine, erythrocyte and platelet replacement requirement decreased in only $13.3 \%(n=2)$ of the patients. Grade 3 and above hematological toxicity was observed in 14 patients. The most common non-hematologic toxicities were fatigue-anorexia (80\%), pneumonia (25\%), and associated with the gastrointestinal tract (40\% nausea and vomiting, 13\% diarrhea). $66.6 \%$ of the patients were complicated with infection. Two patients had COVID-19 infection and recovered with treatment. TLS was developed in 2 patients. One of these patients had TLS at the time of diagnosis. Venetoclax dose reduction or an interruption was required in $60 \%(n=9)$ patients. The mortality rate in the first 30 days was $20 \%$.

The management of AML gradually turns into an individualized approach to applying targeted therapies. The time to enter early remission is critical. For this reason, deaths due to infection should be prevented. Although the toxicities of this combination therapy can be managed, close follow-up of the patients is mandatory.
Venetoclax+azacytidine combination therapy is relatively well-tolerated, can improve blood counts, relieve symptoms, improve life quality, and prolong survival. Still, more studies are needed to assess long-term disease control.

\section{Conflict of Interests}

Authors declare that there are none.

\section{Acknowledgment}

This study has been presented in $17^{\text {th }}$ Uludag Internal Medicine National Winter Congress, $6^{\text {th }}$ Bursa Family Medicine Association National Congress, $11^{\text {th }}$ Uludag Internal Medicine Nursing Congress, 5-7 March 2021, Bursa, Turkey.

\section{References}

1. Almeida AM, Ramos F. Acute myeloid leukemia in the older adults. Leuk Res Rep. 2016 Jun 16;6:1-7. doi: 10.1016/j.1rr.2016.06.001.

2. Song X, Peng Y, Wang X, Chen Y, Jin L, Yang T, Qian M, Ni W, Tong X, Lan J. Incidence, Survival, and Risk Factors for Adults with Acute Myeloid Leukemia Not Otherwise Specified and Acute Myeloid Leukemia with Recurrent Genetic Abnormalities: Analysis of the Surveillance, Epidemiology, and End Results (SEER) Database, 2001-2013. Acta Haematol. 2018;139(2):115127. doi: $10.1159 / 000486228$.

3. Döhner H, WeisdorfDJ, Bloomfield CD. Acute Myeloid Leukemia. N Engl J Med. 2015 Sep 17;373(12):1136-52. doi: 10.1056/NEJMra1406184.

4. DiNardo CD, Jonas BA, Pullarkat V, Thirman MJ, Garcia JS, Wei AH, Konopleva M, Döhner H, Letai A, Fenaux P, Koller E, Havelange V, Leber B, Esteve J, Wang J, Pejsa V, Hájek R, Porkka K, Illés A, Lavie D, Lemoli RM, Yamamoto K, Yoon SS, Jang JH, Yeh SP, Turgut M, Hong WJ, Zhou Y, Potluri J, Pratz KW. Azacitidine and Venetoclax in Previously Untreated Acute Myeloid Leukemia. N Engl J Med. 2020 Aug 13;383(7):617-629. doi: 10.1056/NEJMoa2012971.

5. DiNardo CD, Pratz K, Pullarkat V, Jonas BA, Arellano M, Becker PS, Frankfurt O, Konopleva M, Wei AH, Kantarjian HM, Xu T, Hong WJ, Chyla B, Potluri J, Pollyea DA, Letai A. Venetoclax combined with decitabine or azacitidine in treatment-naive, elderly patients with acute myeloid leukemia. Blood. 2019 Jan 3;133(1):7-17. doi: 10.1182/blood-2018-08-868752. 